\title{
LAS REVISTAS ELECTRÓNICAS DE PSICOLOGÍA EN EL CONTEXTO DE LOS RECURSOS DE INFORMACIÓN AUTOMATIZADA
}

\author{
Vicente Lázaro Ruiz \\ Eva Iradier Santos \\ Universidad de La Rioja
}

\begin{abstract}
RESUMEN. En los contextos educativos han comenzado a utilizarse masivamente fuentes de información automatizadas. La cantidad de documentos disponibles en la Red genera problemas de búsqueda y selección de recursos informativos en todos los campos del conocimiento científico, también en el de la Psicología. Antes de ofrecer la lista de las revistas electrónicas de Psicología, objetivo principal de este artículo, introducimos al lector en las publicaciones electrónicas y en el contexto general de las revistas electrónicas. En la lista se recogen más de 150 títulos relativos a las seis grandes áreas de la ciencia psicológica. Las cabeceras de las revistas se han ordenado alfabéticamente y en cada una se ha señalado el título, la institución/entidad, el tipo de información en línea, URL y otros tipos de información.

Palabras clave: psicología, publicaciones electrónicas, revistas electrónicas, fuentes de información automatizadas, Internet.
\end{abstract}

ABSTRACT. Nowadays, it has been started to use electronic resources in the education world. The amount of documents that is available in the Net produces problems to search and select electronic resources in every scientific knowledge and in psychological science also. Before showing the electronic publishing list, this is the main aim in the report, we introduce the researcher to electronic journals and to electronic publishing in general. It has been shown more than 150 titles that have relation with the most important six areas in the psychological science. The electronic journals have been arranged in alphabetic order and in everyone it has been market the title, the institution, the sort of online information, the URL and their piece of news.

Keywords: Psychology, electronic publishing, electronic journals, electronic resources, Internet.

\section{Introducción: las publicaciones electrónicas}

Corren tiempos en los que los investigadores y docentes -especialmente si éstc: son del ámbito universitario- se están viendo obligados a modificar sus hábitos de búsqueda y recogida de información referencial para sus tareas laborales cotidianas. Los profesionales de cualquier campo de conocimiento relacionado con el contexto de la educación han comenzado a utilizar decididamente las fuentes de información 
automatizadas. Sin duda, el uso de estas fuentes es más eficaz, rápido, exhaustivo y fácil que los tradicionales sistemas impresos, que continúan siendo válidos.

Los nuevos hábitos socio-culturales de búsqueda y recogida de información han sido producidos fundamentalmente por la Red, por el fenómeno Internet, que permite consultar documentos en web, acceder a catálogos remotos o utilizar bases de datos. No obstante, el manejo del mundo de Internet conlleva un gran problema: la multitud de documentos que están disponibles en la Red. En la búsqueda y selección de sus recursos de mayor interés, se pierde mucho tiempo. Por ello, cada día es más importante contar con guías especializadas de recursos para localizar con facilidad la información que se precisa.

Si rebuscamos referencias bibliográficas acerca de guías especializadas para localizar información, podemos encontrar diversas alternativas como la de Martínez López y otros (1997); o la de Morville, Rosenfeld y Janes (1996). Nosotros mismos (Lázaro e Iradier, en presâ) esbozamos una tipología acerca de los recursos informativos automatizados. En ella propusimos clasificar las fuentes de información distinguiendo cuatro grandes grupos: 1) catálogos (información bibliográfica), 2) bases de datos, 3) foros de discusión y 4) publicaciones electrónicas. Precisamente abordamos aquí estas últimas.

Con el nombre de publicaciones electrónicas se hace referencia a los textos y multimedia que tienen en común que son aportaciones hechas para su difusión masiva y, además, que esas aportaciones pueden considerarse publicadas/autentificádas y accesibles por medios electrónicos.

En una primera aproximación, podemos encuadrar las publicaciones electrónicas en cuatro grandes bloques: 1) publicaciones unitarias, 2) artículos sueltos a texto completo, 3) actas de las ponencias de congresos, y 4) artículos publicados en revistas electrónicas. A continuación nos referiremos a algunas características detectadas en los cuatro grupos de publicaciones, aunque no lo haremos de manera sistemática.

Dentro de las publicaciones unitarias podemos encontrar al menos dos grandes apartados: las monografías y las obras de referencia. Las monografías, quizás porque su estructura física no se presta a que sean consultadas en la pantalla del ordenador, no abundan en la Red. Por el contrario, como señala Baró (1997), las obras de referencia (diccionarios, enciclopedias, repertorios de legislación o jurisprudencia, bibliografías y otras) están inundando la Red. Ello es debido, entre otras causas, a la estructura de estas obras que puede parcializarse fácilmente, a la posibilidad de localizar rápidamente la información mediante la introducción de términos de búsqueda, y a la relativa facilidad con la que se actualizan. Todos estos factores hacen que la consulta de las obras de referencia sea más idónea a través de los medios electrónicos que a través del papel impreso.

Los artículos publicados en la Red constituyen a veces verdaderas aportaciones inéditas. Pero ello, por el momento, no suele ser to habitual. En los foros de discusión -listas de distribución y news-, se suelen reproducir artículos publicados previamente de forma impresa o se presentan enlaces cuando los artículos se encuentran en formato electrónico en otros lugares de la Red. Algunas veces, los artículos sueltos a texto completo que aparecen en los foros de discusión son, asimismo, aportaciones originales. 
No hace mucho tiempo, las actas de los congresos tardaban meses en publicarse. Posteriormente, comenzaron a hacerse preimpresiones con las aportaciones presentadas a los congresos. Ahora, además, cada día son más numerosos los foros que ponen en la Red sus conclusiones de forma inmediata.

Antes de pasar a centrarnos en el último de los cuatro grupos, en las publicaciones electrónicas por excelencia, en las revistas electrónicas, queremos indicar que las publicaciones que se difunden a través de las redes telemáticas están planteando nuevos problemas de derechos de autor y de propiedad intelectual y, también, el de considerar cuándo una obra introducida en Internet está publicada, dado que la distribución no implica en sí la publicación, como señalan Barrueco y otros (1996)

\section{Las revistas electrónicas}

Las redes telemáticas están posibilitando un cambio revolucionario en el medio de transmisión del trabajo científico por excelencia: la publicación periódica. El desarrollo de las revistas en formato electrónico ha sido vertiginoso desde su aparición. Las revistas electrónicas accesibles en Internet son cada día más abundantes. Las editoras y distribuidoras de revistas impresas están ampliando su campo de acción al de las revistas electrónicas y ofrecen con frecuencia nuevos títulos y nuevos servicios.

El aumento de la importancia y del número de revistas electrónicas se refleja en la aparición de programas para favorecer el acceso y el desarrollo de este tipo de recursos electrónicos. En nuestro país podemos señalar, a modo de ejemplo, los programas Decomate; así como las actividades del Grupo Español de Revistas. Decomate es un programa para el desarrollo de investigaciones sobre el acceso a revistas electrónicas a texto completo en el que colaboran las Universidades de Tilburg (Hoianda), la Autónoma de Barcelona y la London School of Economics. El 1 de febrero de 1998 entraba en funcionamiento el programa Decomate II : Developing the European Digital Library for Economics, centrado en el campo de la Economía «http://decomate.uab.es"

En la línea anterior de preocupación por el tema encontramos al Grupo Español de Revistas (G.E.R.), que está formado por bibliotecarios, editores, distribuidores y otros sectores inmersos en el mundo de las publicaciones periódicas. Este Grupo celebró los pasados 9 y 10 de octubre su Reunión Anual en Granada. El tema central de esta reunión de 1997 fue precisamente la edición electrónica de publicaciones periódicas.

Vamos a hacernos eco de la revista electrónica, deteniéndonos en la concepción del término, en las razones de su rápido crecimiento, en la localización de las revistas, y en los tipos de información que se pueden encontrar en la Red sobre revistas.

El término revista electrónica -electronic journal-es ambiguo. Covi y Kling (1996) nos dicen que no está claro si el término se refiere al formato de distribución de una revista impresa, al archivo electrónico de números atrasados de la revista impresa o a una revista publicada exclusivamente en un formato electrónico. Para Barrueco y otros (1996, pág. 306), las revistas electrónicas son "aquel conjunto de artículos ordenados, formalizados y publicados bajo la responsabilidad de una institución científica o técnica a través de redes teleinformáticas". Algunos de los autores anteriores creen que "una revista electrónica no es una versión en formato electrónico de una 
revista en papel, sino que debe ser concebida desde el comienzo hasta el fin en formato electrónico" (Barrueco y García Testal, 1997, pág. 19). Algunos autores, como Franks (1993) y Woodward y McKnigth (1995) admiten que también puede denominarse revistas electrónicas a aquellas que, sin ser a texto completo, nos permiten acceder a algunos artículos completos, a resúmenes o simplemente a las tablas de contenido. Incluyen también el formato en soporte digital (CD-ROM).

Nosotros nos atreveríamos a decir que la peculiaridad de la revista electrónica está en el medio de transmisión de la información, que ha de ser automatizado. Incluimos aquí el soporte digital. Además, integraríamos en la concepción del término cualquier tipo de acceso a la información contenida en una revista: 1) las informaciones editoriales, 2) el acceso a los sumarios, 3) el acceso a resúmenes de los artículos y 4) los artículos a texto completo.

En cuanto al crecimiento que están experimentando las revistas electrónicas en la Red, pensamos que puede explicarse por estas cuatro razones: 1) por la reducción de los costes de producción y de distribución que suponen para los editores; 2) por la posibilidad de interacción que ofrecen entre autores y lectores; 3) por la amplia gama de posibilidades de presentación y diseño que permiten, al poderse incorporar elementos multimedia como el audio, el vídeo o el software incorporado (Fecko, 1997); y 4) por la rapidez que permiten para difundir la información científica.

Como medio de difusión, la revista científica es el principal vehículo de transmisión de los conocimientos y avances científicos, sobre todo en el ámbito de las disciplinas científico-técnicas, en las que la información tiene un mayor nivel de obsolescencia que en las ciencias humanas y sociales. A la rapidez en la difusión de la información que tienen de por sí las publicaciones periódicas se une la inmediatez del acceso electrónico.

Para la localización de revistas electrónicas existen diversos medios (desde los buscadores conocidos de Internet hasta los directorios específicos de revistas), que podemos agrupar en directorios de carácter general y directorios especializados de Psicología, en el caso que nos ocupa. De entre los directorios de carácter general podemos señalar, a modo de ejemplo, y basándonos en la información que ofrece la sede web de la Biblioteca Universitaria de la Universidad de La Rioja «http://www.unirioja.es/biblio/BIB-MAIN.HTM» :

- Australian Electronic Journals, National Library of Australia «http://www.nla.gov.au/oz/ausejour.html»

- CIC Electronic Journal Collection, The "http://ejournals.cic.net»

- Electronic Journal Access, Colorado Alliance of Research Libraries (ARL) "http://arl.cni.org/scomm/edir»

- Electronic Journals, University of Pennsylvania Library "http://www.library.upenn.edu/resources/ej/xej-news-index.html»

- JSTOR Project, The «http: //jstor.umdl.umich.edu/»

- NewJour, University of California, San Diego «http://gort.ucsd.edu/newjour»

- OCLC : Electronics Collections Online «http://www.oclc.org/oclc/eco/publish.htm» 
- Scholary Journals distributed via the World-Wide-Web, University of Houston Libraries

"http://info.lib.uh.edu/wj/webjour.html»

Directorios de recursos especializados en Psicología en los que se pueden encontrar revistas electrónicas, entre los muchos que existen:

- American Psychological Association "http://www.apa.org/journals/»

- British Psychological Society "http://journals.eecs.qub.ac.uk/BPS/BritishPsychological.html»

- Colegio Oficial de Psicólogos (Madrid) «http: //www.cop.es/publi.htm»

- Hanover College «http: //psych.hanover.edu/Krantz/journal.html»

- Psychology Press Journals (UK) «http://www.tandf.co.uk/psypress/mainjnls.htm»

- Universidad Autónoma de Madrid. Biblioteca Universitaria. "http://www.uam.es/estructura/servicios/Bibliotecas/paginas/psicolo.html»

- Universidad Complutense de Madrid. Biblioteca de la Fac. de Psicología. "http://www.ucm.es/BUCM/psi/0500.htm»

Para finalizar este apartado, nos referimos al tipo de información que podemos encontrar en la Red sobre revistas. Existen diferentes formas de acceso a su información: 1) Servidores que contienen información general sobre una determinada publicación: datos sobre el editor, direcciones y teléfonos, consejo de redacción y consejo editorial, colaboradores, servicios de acceso electrónico y suscripción, línea editorial, periodicidad, números normalizados (ISSN y Depósito Legal). 2) Servidores que, además de la información general, contienen los sumarios del/os último/s número/s de la revista, o bien de la revista completa. A veces estos servidores presentan enlaces a resúmenes de los artículos. 3) Revistas a texto completo, que presentan los sumarios con enlaces al artículo desarrollado en su totalidad, de forma gratuita o por suscripción. 4) Sistemas de acceso a los sumarios de las revistas a través de bases de datos comerciales (Uncover), o a través de la propia institución que edita la revista o boletín, como la American Psychological Association, que lo hace mediante PsychNet «http://baldwin.apa.org:88/plweb-cgi/fastweb?searchform+apa». La base de datos puede contener información sobre una revista, sobre toda la información contenida en el servidor, sobre todas las revistas editadas por una institución o bien sobre miles de publicaciones periódicas -en el caso de las grandes bases de datos comerciales-. 5) Servicios que mantienen las grandes empresas distribuidoras de acceso a sumarios de revistas -generalmente por suscripción- y suministro de los artículos vía correo convencional, fax o correo electrónico. Existen otros servicios, denominados genéricamente CAS-IAS (Current Awareness Service-Individual Article Supply), que proporcionan las mismas ventajas que los anteriormente citados, pero que además se constituyen como servicios de alerta dirigidos al usuario final (Peña, 1995)

Así por ejemplo, Swets («http://www.swets.nl») mantiene servicios como SwetScan, que es un servicio de alerta y suministro de artículos a través de Internet o como SwetsNet, que permite a los suscriptores el acceso electrónico al texto comple- 
to de los artículos de numerosas revistas, al catálogo de todas las publicaciones periódicas de Swets y a las búsquedas en sumarios y resúmenes. SwetsNet es, en suma, un servicio diseñado para su utilización "a la carta" en bibliotecas o centros con un gran número de accesos y suscripciones. En estos momentos, la Universidad de La Rioja mantiene a prueba la suscripción al servicio para sus usuarios. SwetsNet permite hacer búsquedas en los sumarios disponibles de las revistas extranjeras suscritas en papel con Swets y acceder a la base de datos completa de Swets, que incluye con 13.000 títulos. Cuenta para ello con 8 passwords y un cupo de 25 sumarios para recibir por correo electrónico por cada password de usuario. Para más información, «http: //www.unirioja.es/biblio/swetsnet.html».

Existen otros servicios con unas características muy similares a las explicadas sobre Swets y, sobre todo, con los mismos fines. Quizás los más importantes sean:

- BIDS de Blackwell «http://www.journalsonline.bids.com/JournalsOnline»

- Cambridge University Press «http://www.cup.org/Journals/JNLSAZ.html»

- EbscoNet de Ebsco "http://www.ebsco.com/ebsconet»

- IDEAL de Academic Press «http://www.apnet.com/www/journal/journals.htm»

- LINK de Springer-Verlag "http://link.springer.de/search.htm»

- Psychology Online de Psychology Press Journals "http://www.tandf.co.uk/psypress/psychonline.htm\#PsychOnline»

- ScienceDirect «http://www.sciencedirect.com» y Elsevier Electronic Subscriptions (EES) "http://www.elsevier.com/locate/ees/» de Elsevier Science.

- Uncover Reveal, que es un servicio de alerta «http:/uncweb.carl.org/reveal». La Biblioteca Universitaria de La Rioja tiene suscrito a prueba este servicio y se realiza a través de perfiles de búsqueda que los usuarios pueden hacer individualmente «http://www.unirioja.es/biblio/UNCOVER.HTM».

\section{Revistas electrónicas de psicología}

Dejamos ahora a un lado algunas otras cuestiones más que las expuestas, relacionadas con las revistas electrónicas en general, para pasar a presentar una relación aproximada de las revistas de Psicología accesibles de forma automatizada a través de los recursos World Wide Web (WWW). Somos conscientes de la dificultad para alcanzar la exhaustividad compilatoria sobre un tema cuando éste está relacionado con Internet, pero lo hemos intentado con el fin de escribir estas páginas.

Creemos que en estas 152 referencias de revistas electrónicas de Psicología está lo más relevante sobre este campo de conocimiento. Creemos, también, que resultará útil para todos los profesionales relacionados con el contexto educativo. En especial lo será tanto para los investigadores e interesados por la Psicología como para los profesionales de la Información y Documentación. 
En nuestra revisión hemos constatado que no hay gran cantidad de revistas electrónicas de Psicología a texto completo, pero sí información a través del acceso a los sumarios o de los servicios que ofrecen las distribuidoras de publicaciones periódicas y de las bases de datos.

Esta lista incluye revistas españolas y extranjeras y revistas de las seis grandes áreas de conocimiento de la ciencia psicológica. Al confeccionarla hemos seguido una guía de normas clasificatorias. Se ha ordenado alfabéticamente y en cada una se ha señalado, si se daba el caso: 1) El título y subtítulos completos. 2) La institución o entidad editora cuando no es comercial. 3) El tipo de información que se ofrece en línea, sea gratuita o no: información general; acceso a sumarios o tablas de contenido; resúmenes de cada artículo; artículos a texto completo. 4) URL de acceso directo. 5) Otro tipo de observaciones, como si permite búsquedas o constituye bases de datos.

Un apunte final antes de pasar a ofrecer la lista, hemos adoptado intencionadamente el formato de lista de revistas con su enlace directo. Con ello hemos pretendido constituir un documento fuente, como lo denomina Codina (1997), disponible en «http://www.unirioja.es/biblio/REVELECT.HTM». Los enlaces estaban activos a 6 de abril de 1998.

- Aging, Neuropsychology and Cognition (Información, sumarios y resúmenes) «http://www.swets.nl/sps/journals/a\%26c.html»

- American Journal of Psychology (Sumarios) «http://mambo.ucsc.edu/psl/ajp/1073.html»

- American Psychologist (American Psychological Association) (Información, sumarios, resúmenes y algún artículo seleccionado a texto completo) «http://www.apa.org/journals/amp.html»

- Anales de Psicología (Universidad de Murcia) (Información y sumarios) «http://www.um.es/ facpsi/public/anales.htm»

- Anuario de Psicología (Universidad de Barcelona) (Información y sumarios) «http://www.ub.es/psicolog/anuari/anuari.html»

- Anuario de Psicología Jurídica (Colegio Oficial de Psicólogos) (Información y sumarios) «http://www.cop.es/publicaciones/juridica/juridica.htm»

- APA Monitor (Búsquedas temáticas a texto completo) «http: //www.apa.org/ monitor/»

- Applied and Preventive Psychology (Información y sumarios) «http://www.cup.org/journals/jnlscat/app/app.html»

- Applied Psycholinguistics (Información y sumarios) «http://www.cup.org/journals/jnlscat/aps/aps.html»

- Applied Psychology (International Association of Applied Psychology) Disponible a texto completo a través de Psychology Online, un base de datos a la que se accede por suscripción «http://www.tandf.co.uk/psypress/psychonline.htm\#PsychOnline» (Información) «http://www.tandf.co.uk/psypress/JNLFILES/APPLPSY.HTM»

- Apuntes de psicología (Colegio Oficial de Psicólogos. Andalucía Occidental) (Información) «http://www.cop.es/publicaciones/apuntes/info.htm»

- Behavior Therapy : an international journal devoted to the application of behavioral and cognitive sciences to clinical problems (Información, sumarios y resúmenes) «http://server.psyc.vt.edu/aabt/bt/home» 
- Behavioral and Brain Sciences: target articles preprints (Información y base de datos para búsqueda de artículos en línea. Comentarios y aportaciones a los artículos por especialistas en la materia) "http://www.cogsci.soton.ac.uk/bbs/»

- Behavioral Neuroscience (American Psychological Association) (Información, sumarios y resúmenes) "http://www.apa.org/journals/bne.html»

- Behavioural and Cognitive Psychotherapy (Información y sumarios) " http://www.cup.org/journals/jnlscat/bcp/bcp.html»

- Brain (Información, sumarios, resúmenes y a texto completo desde diciembre 1997) «http://www.oup.co.uk/brainj/contents/»

- Brain and Cognition: journal of clinical, experimental and theoretical research (Información y sumarios) "http://www.apnet.com/www/journal/br.htm»

- Brain and language (Información y sumarios) "http://192.215.52.3/www/journal/bl.htm»

- Bristish Journal of Clinical Psychology, The (British Psychological Society) (Información y sumarios. Servicio de alerta y a texto completo por suscripción) «http://journals.eecs.qub.ac.uk/BPS/BJCP/BJCP.html»

- British Journal of Developmental Psychology, The (British Psychological Society) (Información y sumarios. Servicio de alerta y a texto completo por suscripción) «http://journals.eecs.qub.ac.uk/BPS/BJDP/BJDP.html»

- British Journal of Educational Psychology, The (British Psychological Society) (Información y sumarios. Servicio de alerta y a texto completo por suscripción) «http://journals.eecs.qub.ac.uk/BPS/BJEP/BJEP.html»

- British Journal of Health Psychology, The (British Psychological Society) (Información y sumarios. Servicio de alerta y a texto completo por suscripción) «http: //journals.eecs.qub.ac.uk/BPS/BJHP/BJHP.html»

- British Journal of Mathematical and Statistical Psychology, The (British Psychological Society) (Información y sumarios. Servicio de alerta y a texto completo por suscripción) «http://journals.eecs.qub.ac.uk/BPS/BJMSP/BJMSP.html»

- British Journal of Medical Psychology, The (British Psychological Society) (Información y sumarios. Servicio de alerta y a texto completo por suscripción) «http://journals.eecs.qub.ac.uk/BPS/BJMP/BJMP.html»

- British Journal of Psychology, The (British Psychological Society) (Información y sumarios. Servicio de alerta y a texto completo por suscripción) "http://journals.eecs.qub.ac.uk/BPS/BJP/BJP.html»

- British Journal of Social Psychology, The (British Psychological Society) (Información, sumarios. Servicio de alerta y a texto completo por suscripción) «http://journals.eecs.qub.ac.uk/BPS/BJSP/BJSP.html»

- Cadernos de Psicoloxia (Colegio Oficial de Psicólogos. Galicia) (Información) «http://www.cop.es/delegaci/galicia/cadernos.html»

- Child Neuropsychology (Información, sumarios y resúmenes) «http://www.swets.nl/sps/journals/child.html»

- Child Psychology and Psychiatry Review (Información y sumarios) uhttp://www.cup.org/journals/jnlscat/cpr/cpr.html»

- Ciencia psicológica (Colegio Oficial de Psicólogos. Extremadura) (Información y sumarios) "http://www.cop.es/publicaciones/cpsicolo/» 
- Clínica y salud: revista de psicología clínica y de salud (Colegio Oficial de Psicólogos) (Información y sumarios) «http://www.cop.es/publicaciones/clinica/clinica.htm»

- Clinical Neuropsychologist, The (Información, sumarios y resúmenes) «http://www.swets.nl/sps/journals/tcn.html»

- Cognition and Emotion. Disponible a texto completo a través de Psychology Online, una base de datos a la que se accede por suscripción. «http://www.tandf.co.uk/psypress/psychonline.htm\#PsychOnline» (Información) «http://www.tandf.co.uk/psypress/jnlfiles/cogem.htm»

- Cognitive and Behavioral Practice (Información, sumarios y resúmenes) «http://server.psyc.vt.edu/aabt/cbp/home»

- Cognitive Neuropsychology. Disponible a texto completo a través de Psychology Online, una base de datos a la que se accede por suscripción. "http://www.tandf.co.uk/psypress/psychonline.htm\#PsychOnline» (Información) «http://www.tandf.co.uk/psypress/JNLFILES/COGNPSYO.HTM»

- Cognitive Psychology (Información y sumarios) «http://apnet.com/www/journal/cg.htm»

- Cognitive Therapy and Research (Información y sumarios. Anuncia texto completo desde el primer número de 1998) «http://www.sci.sdsu.edu/CAL/CTR/CTR.html»

- Community and Applied Social Psychology (Información) «http://www.wiley.com/journals/casp/»

- Consciousness and Cognition (Association for the Scientific Study of Consciousness) (Información) «http://192.215.52.3/www/catalog/cc.htm»

- Consulting Psychology Journal : practice and research (American Psychological Association) (Información, sumarios y resúmenes) «http://www.apa.org/journals/cpb.html»

- Contemporary Educational Psychology (Información y sumarios. Presenta un foro para la discusión sobre los artículos a publicar) "http://www.apnet.com/www/journal/ep.htm»

- Contemporary Psychology (American Psychological Association) (Información y sumarios) «http://www.apa.org/journals/cnt.html»

- Current Directions in Psychological Science (Información y sumarios) «http: //www.cup.org/journals/jnlscat/cdp/cdp.html»

- Current Research in Social Psychology (University of lowa) (Información, sumarios y texto completo) «http://www.uiowa.edu/ grpproc/crisp/crisp.html»

- Development Psychology (American Psychological Association) (Información, sumarios y resúmenes) «http://www.apa.org/journals/dev.html»

- Development and Psychopathology (información y sumarios) «http ://www.cup.org/journals/jnlscat/dpp/dpp.html»

- Developmental Review (Información y sumarios) «http://www.apnet.com/www/journal/dr.htm»

- Documentación psicológica : revista de sumarios (Colegio Oficial de Psicólogos) (Información) «http: //www.cop.es/publicaciones/documenta/documen.htm»

- Ecological Psychology (Información y sumarios) «http://www.trincoll.edu/ psyc/journal.html» 
- Education Policy Analysis Archives: a refereed journal of the American Education Research Association (Información, sumarios y resúmenes) "http://olam.ed.asu.edu/epaa/»

- Educational Psychology Review (The University of Texas) (Información, sumarios y texto completo) "http://www.edb.utexas.edu/edpreview/»

- Electronic Journal of Cognitive and Brain Sciences, The (Información incompleta, aunque se anuncia como una revista electrónica de libre acceso) «http://osiris.rutgers.edu/ zoli/ejcbs_letter.html»

- Enginy (Colegio Oficial de Psicólogos. Baleares) (Información y sumarios) "http://www.cop.es/publicaciones/enginy"

- European Journal of Cognitive Psychology. Disponible a texto completo a través de Psychology Online, un base de datos a la que se accede por suscripción. «http://www.tandf.co.uk/psypress/psychonline.htm\#PsychOnline») (Información) «http:// www.tandf.co.uk/psypress/JNLFILES/EUCOGPSY.HTM»

- European Journal of Psychological Assessment (European Association of Psychological Asessment) (Información y sumarios) "http://www.uam.es/estructura/facultades/Psicologia/paginas/eapa/ejpa.html»

- European Journal of Work and Organizational Psychology (Association of Work and Organizational Psychology) Disponible a texto completo a través de Psychology Online, un base de datos a la que se accede por suscripción. «http://www.tandf.co.uk/psypress/psychonline.htm\#PsychOnline») (Información) «http: //www.tandf.co.uk/psypress/JNLFILES/EUWOPSY.HTM»

- European Psychologist (APA-EFPPA) (Información, sumarios y resúmenes) «http://www.apa.org/journals/epp.html»

- Experimental and Clinical Psychopharmacology (American Psychological Association) (Información, sumarios y resúmenes) «http://www.apa.org/journals/pha.html»

- Group dinamics: theory, research and practice (American Psychological Association) (Información, sumarios y resúmenes) whttp://www.apa.org/journals/gdn.html»

- Harvard Brain, The (Harvard University Undergraduate Journal of Neuroscience) (Información, sumarios y texto completo) «http://hcs.harvard.edu/ husn/BRAIN/index.html»

- Health Psychology (American Psychological Association) (Información, sumarios y resúmenes) «http://www.apa.org/journals/hea.html»

- History of Psychology (American Psychological Association) (Información, sumarios y resúmenes) «http://www.apa.org/journals/hop.html»

- Iberpsicología : Anales de la Revista de Psicología General y Aplicada. Publicación electrónica de la Federación Española de Asociaciones de Psicología y la U.C.M. (Información, sumarios y texto completo) «http://fs-morente.filol.ucm.es/publicaciones/lberpsicologia/lberpsicologia.htm

- Infancia y aprendizaje (Información, sumarios y resúmenes) «http://www.ub.es/psicolog/infancia/inf_apr.html»

- Informació psicológica (Colegio Oficial de Psicólogos. Valencia) (Sumarios) "http://www.cop.es/publicaciones/informacio/» 
- International Journal of Behavioural Development (International Society for the Study of Behavioural Development). Disponible a texto completo a través de Psychology Online, un base de datos a la que se accede por suscripción. «http://www.tandf.co.uk/psypress/psychonline.htm\#PsychOnline») (Información) «http:/www.tandf.co.uk/psypress/JNLFILES/IJBEHDEV.HTM»

- International Journal of Neuro-psychopharmachology (Información y sumarios para 1998) «http://www.cup.org/journals/jnlscat/pnp/pnp.html»

- International Journal of Psychology. Disponible a texto completo a través de Psychology Online, un base de datos a la que se accede por suscripción. «http://www.tandf.co.uk/psypress/psychonline.htm\#PsychOnline») (Información) «http:/www.tandf.co.uk/psypress/JNLFILES/IJOPSYO.HTM»

- International Journal of Psychopathology, Psychopharmacologie and Psychotherapy (Información y texto completo) «http://www.psycom.net/ijppp.html»

- Intervención psicosocial : revista sobre igualdad y calidad de vida (Colegio Oficial de Psicólogos) (Información y sumarios) "http://www.cop.es/publicaciones/psicosocial/psicosocial.htm»

- Journal of Abnormal Psychology (American Psychological Association) (Información, sumarios y resúmenes) «http: //www.apa.org/journals/abn.html»

- Journal of Adolescence (Información y sumarios) «http://www.hbuk.co.uk/ap/journals/ad/»

- Journal of the American Psychoanalytic Association, The (Información, sumarios y resúmenes) «http://apsa.org/japa/index.htm»

- Journal of Applied Behavior Analysis (Información, sumarios y un artículo a texto completo por número, desde 1995. Permite búsquedas en títulos y resúmenes)

«http://www.envmed.rochester.edu/wwwrap/behavior/jaba/jabahome.htm»

- Journal of Applied Psychology (American Psychological Association) (Información, sumarios y resúmenes) «http:/www.apa.org/journals/apl.html»

- Journal of Child Psychology and Psychiatry and allied disciplines, The (Información y sumarios) «http://www.cup.org/journals/jnlscat/cpp/cpp.html»

- journal of Clinical and Experimental Psychology (Información, sumarios y resúmenes) «http://www.swets.nl/sps/journals/jcen.html»

- Journal of Comparative Neuropsychology (American Psychological Association) (Información, sumarios y resúmenes) «http://www.apa.org/journals/com.html»

- Journal of Constructivist Psychology (Sumarios) «http://ksi.cpsc.ucalgary.ca/PCP/JCP95.html»

- Journal of Consulting and Clinical Psychology (American Psychological Association) (Información, sumarios y resúmenes) «http://www.apa.org/journals/ccp.html»

- Journal of Counseling Psychology (American Psychological Association) (Información, sumarios y resúmenes) «http://www.apa.org/journals/cou.html»

- Journal of Credibility Assessment and Witness Psychology (Boise State University) (Información, resúmenes y texto completo) «http://truth.idbsu.edu/jcaawp/default.html» 
- Journal of Cross-Cultural Psychology (Información y sumarios) «http://www.fit.edu/CampusLife/clubs-org/iaccp/JCCP/jccp.htm/»

- Journal of Educational Psychology (American Psychological Association) (Información, sumarios y resúmenes) «http://www.apa.org/journals/edu.html»

- Journal of Environmental Psychology (Información y sumarios) «http://www.hbuk.co.uk/ap/journals/ps/»

- Journal of the Experimental Analysis of Behavior (Información, sumarios y un artículo a texto completo por número desde 1995. Permite búsquedas en resúmenes y títulos)

«http://www.envmed.rochester.edu/wwwrap/behavior/jeab/jeabhome.htm»

- Journal of Experimental Child Psychology (Información y sumarios) "http://www.apnet.com/www/journal/ch.htm»

- Journal of Experimental Psychology: Animal Behavior Processes (American Psychological Association) (Información, sumarios y resúmenes) «http://www.apa.org/journals/xan.html»

- Journal of Experimental Psychology: Applied (American Psychological Association) (Información, sumarios y resúmenes) "http://www.apa.org/journals/xap.html"

- Journal of Experimental Psychology: General (American Psychological Association) (Información, sumarios y resúmenes) «http: //www.apa.org/journals/xge.html»

- Journal of Experimental Psychology: Human perception and Performance (American Psychological Association) (Información, sumarios y resúmenes) «http://www.apa.org/journals/xhp.html»

- Journal of Experimental Psychology: Learning, Memory and Cognition (American Psychological Association) (Información, sumarios y resúmenes) «http://www.apa.org/journals/xim.html»

- Journal of Experimental Social Psychology (Información y sumarios) «http://www.apnet.com/www/journal/js.htm»

- Journal of Family Psychology (American Psychological Association) (Información, sumarios y resúmenes) «http://www.apa.org/journals/fam.html»

- Journal of Health Psychology (Información y sumarios) «http://www.mdx.ac.uk/www/jhp/index.htmlx»

- Journal of the Learning Sciences, The (Información) «http://www.cc.gatech.edu/aimosaic/faculty/kolodner/jls/»

- Journal of Mathematical Psychology (Información y sumarios) «http://www.apnet.com/www/journal/mp.htm»

- Journal of Memory and Language (Información y sumarios) «http://www.apnet.com/www/journal/ml.htm»

- Journal of Mind and Behavior, The (Información, sumarios y resúmenes. Permite búsquedas en los resúmenes) «http://kramer.ume.maine.edu/ jmb/welcome.html»

- Journal of Occupational Health Psychology (American Psychological Association) (Información, sumarios y resúmenes) «http://www.apa.org/journals/ohp.html»

- Journal of Occupational and Organizational Psychology, The (British Psychological Society) (Información y sumarios. Servicio de alerta y a texto completo por suscripción)

«http://journals.eecs.qub.ac.uk/BPS/JOOP/JOOP.html» 
- Journal of Parenting Reasearch (Boise State University) (Información y sumarios) «http://truth.idbsu.edu/jpr/default.html»

- Journal of Personality and Social Psychology (American Psychological Association) (Información, sumarios y resúmenes) «http://www.apa.org/journals/psp.html»

- Journal of Psychology and the Behavioral Sciences (Información, sumarios y resúmenes) "http://alpha.fdu.edu/psychweb/JPBS.htm»

- Journal of Research in Personality (Información y sumarios) "http://www.apnet.com/www/journal/rp.htm»

- Journal of Rural Community Psychology (Información, sumarios y texto completo): «http://www.marshall.edu/jrcp/index.html»

- Journal of Theoretical and Philosophical Psychology (American Psychological Association) (Información y sumarios) «http://www.yorku.ca/dept/psych/orgs/apa24/journal.htm»

- Language and Cognitive Processes. Disponible a texto completo a través de Psychology Online, un base de datos a la que se accede por suscripción. «http://www.tandf.co.uk/psypress/psychonline.htm\#PsychOnline») (Información) "http://www.tandf.co.uk/psypress/3NLFILES/langcogp.htm»

- Laterality. Disponible a texto completo a través de Psychology Online, un base de datos a la que se accede por suscripción.

"http://www.tandf.co.uk/psypress/psychonline.htm\#PsychOnline» (Información) «http: //www.tandf.co.uk/psypress/JNLFILES/LATERAL.HTM»

- Learning and Motivation (Información y sumarios) «http://www.apnet.com/www/journal//m.htm»

- Legal and Criminological Psychology (British Psychological Society) (Información y sumarios. Servicio de alerta y a texto completo por suscripción) «http://journals.eecs.qub.ac.uk/BPS/LCP/LCP.html»

- Man, neuron, model ; Email Communications in Psychophysiology (Association of Psychophysiologist) (Información, sumarios y texto completo) «http://www.hku.hk/psycho/abstracts1.html»

- Mathematical Cognition. Disponible a texto completo a través de Psychology Online, un base de datos a la que se accede por suscripción.

«http://www.tandf.co.uk/psypress/psychonline.htm\#PsychOnline» (Información) «http://www.tandf.co.uk/psypress/JNLFILES/MATHCOG.HTM»

- Memory : Cognitive, Clinical, Developmental, Educational, Neuropsychological, and Social. Disponible a texto completo a través de Psychology Online, un base de datos a la que se accede por suscripción.

«http://www.tandf.co.uk/psypress/psychonline.htm\#PsychOnline» (Información) «http://www.tandf.co.uk/psypress/JNLFILES/MEMORY.HTM»

- Neuropsychologyical Rehabilitation. Disponible a texto completo a través de Psychology Online, un base de datos a la que se accede por suscripción. «http://www.tandf.co.uk/psypress/psychonline.htm\#PsychOnline» (Información) "http://www.tandf.co.uk/psypress/JNLFILES/NEUREHAB.HTM»

- Neuropsychology (American Psychological Association) (Información, sumarios y resúmenes) "http://www.apa.org/journals/neu.html"

- Organizational Behavior and Human Decisions Processes (Información y sumarios) "http://www.apnet.com/www/journal/ob.htm»

- Papeles del psicólogo (Coiegio Oficial de Psicólogos) (Información, con algún artículo a texto completo) «http://www.cop.es/publicaciones/papeles/» 
El suplemento INFOCOP aparece a texto completo «http://www.cop.es/papeles/papeles_infocop68.htm»

- Professional Psychology: research and practice (American Psychological Association) (Información, sumarios y resúmenes) «http://www.apa.org/journals/pro.html»

- Psicología.com: revista electrónica de Psicología (Información, sumarios y texto completo) «http: //www.psiquiatria.com/psicologia/»

- Psychoanalytic Psychology: a journal of Theory, practice, research and criticism (APA) (Información, sumarios y resúmenes) «http://www.apa.org/journals/pap.html»

- Psychology Software News (The University of York, U.K.) (Información y sumarios) "http://www.york.ac.uk/inst/ctipsych/web/CTI/CTIInfo-PSN.html»

- Psychological Assessment (American Psychological Association) (Información, sumarios y resúmenes) «http://www.apa.org/journals/pas.html»

- Psychological Bulletin (American Psychological Association) (Información, sumarios y resúmenes) «http://www.apa.org/journals/bul.html»

- Psychological Methods (American Psychological Association) (Información, sumarios y resúmenes) "http://www.apa.org/journals/met.html»

- Psychological Research: an international journal of perception, attention, memory and action. (Información, sumarios, resúmenes y texto completo) Editada por Springer, acceso por suscripción a través del servicio LINK. Existe versión impresa.

«http://link.springer.de/link/service/journals/00426/index.htm»

- Psychological Review (American Psychological Association) (Información, sumarios y resúmenes) «http://www.apa.org/journals/rev.html»

- Psychology and Aging (American Psychological Association) (Información, sumarios y resúmenes) «http://www.apa.org/journals/pag.html»

- Psychology of Women Quarterly (Información y sumarios) «http://www.cup.org/journals/jnlscat/pwq/pwq.html»

- Psychology, Public policy and Law (American Psychological Association) (Información, sumarios y resúmenes) «http: //www.apa.org/journals/law.html»

- Psychophysiology (Información y sumarios) "http://www.cup.org/journals/jnlscat/pgy/pgy.html»

- Psycoloquy : a refereed international, interdisciplinary electronic journal sponsored by the APA. Publishes target articles and peer commentary in all areas of psychology as well as cognitive science, neuroscience, behavioral biology, artificial intelligence, robotics/vision, linguistics and philosophy. (Información, sumarios y texto completo. Se puede recibir por correo electrónico) «http://www.princeton.edu/ harnad/psyc.html"

- Psychometrika : a journal of quantitative psychology (The Psychometric Society) (Información y sumarios) «http://vision.arc.nasa.gov/publications/Psychometrika/»

- Psicothema (Colegio Oficial de Psicólogos) (Información y sumarios) «http://www.cop.es/publicaciones/psycothema/psycho.htm»

- Quarterly Journal of Experimental Psychology: comparative and physiological psychology Disponible a texto completo a través de Psychology Online, un base de datos a la que se accede por suscripción. «http://www.tandf.co.uk/psypress/psychonline.htm\#PsychOnline» 
(Información) «http://www.tandf.co.uk/psypress/JNLFILES/QEXPPSYB.HTM»

- Quarterly Journal of Experimental Psychology: human experimental psychology. Disponible a texto completo a través de Psychology Online, un base de datos a la que se accede por suscripción. «http://www.tandf.co.uk/psypress/psychonline.htm\#PsychOnline» (Información) «http://www.tandf.co.uk/psypress/JNLFILES/QEXPPSYA.HTM»

- Rehabilitation Psychology (American Psychological Association) (Información, sumarios y resúmenes) «http://www.apa.org/journals/rep.html»

- REMA : Revista Electrónica de Metodología Aplicada. Revista Internet de metodología aplicada a la Psicología y Educación. (Universidad de Oviedo) (Información, sumarios y texto completo) «http://www3.uniovi.es/user_html/herrero/REMA/»

- Review of General Psychology (American Psychological Association) (Información, sumarios y resúmenes) «http://www.apa.org/journals/gpr.html»

- Revista de Psicodidáctica (Universidad del País Vasco) (Información, sumarios, resúmenes y algunos artículos a texto completo) «http://www.vc.ehu.es/deppe/revista.html»

- Revista de Psicología del Trabajo y de las Organizaciones (Colegio Oficial de Psicólogos) (Información y sumarios) «http://www.cop.es/publicaciones/trabajo/index.html»

- Revista de Psicología Educativa: la revista de los psicólogos de la educación (COP) (Información y sumarios)

«http://www.cop.es/publicaciones/educativa/reducati.htm»

- Revista de Psicología de la Salud (Universidad Autónoma de Madrid) (Información y sumarios) «http://www.uam.es/estructura/facultades/Psicologia/paginas/psic_salud/default.html»

- Selecciones de prensa (Colegio Oficial de Psicólogos) (Información) «http://www.cop.es/publicaciones/selecciones/prensa.htm»

- Self-help \& Psychology Magazine (Texto completo) "http://www.cybertowers.com/selfhelp/»

- Síntesis psicológica (Colegio Oficial de Psicólogos. Tenerife) (Sumarios) «http://www.cop.es/publicaciones/sintesis/»

- Theory \& Psychology (Información y sumarios) «http://www.psych.ucalgary.ca/thpsyc/»

- Theory and Review in Psychology : a complete refereed e-journal (Texto completo) «http://www.gemstate.net/susan/»

- Thinking and Reasoning. Disponible a texto completo a través de Psychology Online, un base de datos a la que se accede por suscripción. "http://www.tandf.co.uk/psypress/psychonline.htm\#PsychOnline» (Información) «http://www.tandf.co.uk/psypress/JNLFILES/THINKAR.HTM»

- TIP : The Industrial-Organizational Psychologist (SIOP-The Society for Industrial \& Organizational Psychology) (Sumarios y texto completo) «http://www.siop.org/TIPApril98/April98.htm»

- Treatment: a peer reviewed, rapid publication, electronic journal published through a collaboration of the American Psychological Association and the American Psychiatric Association. (Información y texto completo) «http://journals.apa.org/treatment/» 
- Visual Cognition. Disponible a texto completo a través de Psychology Online, un base de datos a la que se accede por suscripción. «http://www.tandf.co.uk/psypress/psychonline.htm\#PsychOnline» (Información) «http://www.tandf.co.uk/psypress/JNLFILES/VISCOG.HTM»

\section{Referencias bibliográficas}

AGUILLO, I. (1998). Hacia un concepto documental de sede web. En IWE, Vol. 7, núm. 1-2, enero-febrero 1998, págs. 45-46

BARO, J. (1997). Obras de referencia en Internet. En IWE, vol. 6, núm. 6, junio 1997, págs. 32-34

BARRUECO, J.M.; GARCÍA TESTAL, C. y GIMENO, M.J. (1996) Una aproximación a las revistas científicas en formato electrónico. En Revista Española de Documentación Científica, vol. 19, núm. 3, págs. 304-313

BARRUECO, J.M. y GARCIA TESTAL, C. (1997). Panorama actual y posibilidades futuras en revistas electrónicas. En IWE, vol. 6, núm. 7, marzo 1997, págs. 18-20

CODINA, LI. (1997) Directorio selectivo de sedes web para documentalistas, estudiosos de los medios de comunicación social y profesionales de la información. En IWE, vol. 6, núm. 9, septiembre 1997, págs. 32-38

COVI, L.M. (1997) The future of electronic journals: unpuzzling researcher's attitudes about electronic journals. En Rev. Esp. De Bib., vol. 1, núm. 1 [Online] Disponible en «http://arcano.lib.surrey.ac.uk/\%7josema/eng/vol1no1/vol1 no1b.html»

COVI, L.M. y KLING, R. (1996) Digital shift or digital drift? : dilemmas of managing digital libary resources in North American Universities [Online] Disponible en «http://hbs.baylor.edu/ramsower/acis/papers/covi.htm»

FECKO, M.B. (1997) Electronic resources : access and issues, London : Bowker Saur

FRANKS, J. (1993) The impact of electronic publishing on scholary journals [Online]. Disponible en «http://cause-www.colorado.edu/information-resources/irlibrary/text/cem9410.txt»

LAZARO, V. e IRADIER, E. (en prensa): Propuesta de un modelo de sistematización de fuentes de información automatizadas en educación a distancia. En I Jornadas sobre la Función Tutorial en la UNED: su presente y su futuro, Madrid, 7-8 noviembre 1997

MARTÍNEZ LÓPEZ, F.J y otros (1997) Internet para investigadores : relación y localización de recursos en la Red para investigadores y universitarios. Huelva : Universidad de Huelva.

MORVILLE, P.; ROSENFELD, L. y JANES, J. (1996) The Internet searcher's handbook : locating information, people \& software. New York : Neal-Shuman

PEÑA, C. de la (1995) Los servicios electrónicos de información puntual y suministro de documentos: revistas frente a artículos. En Revista Española de Documentación Científica, vol. 18, núm. 2, 1995, págs. 188-203

WOODWARD, H. y MCKNIGTH, C. (1995) Electronic journals : issues of access and bibliographical control. En Serials Review, vol. 2, núm. 5, págs. 289-294

Fecha de entrega del original: 7-4-97 


\section{EXPERIENCIAS Y REFLEXIONES}




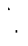

